\title{
Time-Delayed Spread of Viruses in Growing Plaques
}

\author{
Joaquim Fort ${ }^{1}$ and Vicenç Méndez ${ }^{2}$ \\ ${ }^{1}$ Departament de Física, Universitat de Girona, Campus de Montilivi, 17071 Girona, Catalonia, Spain \\ ${ }^{2}$ Facultat de Ciències de la Salut, Universitat Internacional de Catalunya, Gomera, s/n,
} 08190 Sant Cugat del Vallès, Catalonia, Spain

(Received 11 March 2002; published 8 October 2002)

\begin{abstract}
The spread of viruses in growing plaques predicted by classical models is greater than that measured experimentally. There is a widespread belief that this discrepancy is due to biological factors. Here we show that the observed speeds can be satisfactorily predicted by a purely physical model that takes into account the delay time due to virus reproduction inside infected cells. No free or adjustable parameters are used.
\end{abstract}

DOI: $10.1103 /$ PhysRevLett.89.178101

PACS numbers: $87.18 . \mathrm{Hf}$

I. Introduction. - Reaction-diffusion fronts arise in solidification [1], superconductors [2], liquid crystals [3], and combustion [4]. They also appear in ecology [5], archaeology [6], genetics [7], and virology [8]. But time-delayed front speed predictions have been so far compared to observations only in two applications, namely, the Neolithic transition in Europe [6] and the Black Death plague [9]. Up to now, the delay time has never previously been taken into account in predicting the speed of fronts that can be experimentally reproduced in the laboratory. This is done here for the first time. A delay effect is used below to explain the replication and spread of viruses in growing plaques. This work is also relevant to physics, because wave-front solutions are important in many fields where a delay time must also be included [10-12].

We investigate the spreading dynamics of viruses which infect bacteria. It is shown that the present type of models are inadequate as they do not take into account the time delay between the arrival of a virus into a cell and the reproduction of the virus. We extend the present models by including this effect. No free or adjustable parameters are used at all. Experiments independent to those we want to explain are used to determine the values of all parameters. With the values thus obtained, we are able to explain the dependence for the front velocity as a function of the bacterial concentration. This is the first type of evidence for the relevance of delay effects in front propagation experiments which can be repeated in the laboratory.

A plaque is a region of dead (i.e., lysed) cells. It forms due to the adsorption of viruses to host cells, their replication within, and the spread of the new generation after lysis. Many virus phages can be successful only in a medium of growing cells. In such cases, plaques will stop growing after the bacteria reach an stationary phase. On the other hand, for viruses such as T7, it has been observed that plaques grow without bound in a medium containing agar-immobilized, stationary-phase host bacteria [13-15]. We deal with this case. Then, it is possible to measure the speed over a long period, but it does not agree with that predicted by existing models [16-18].

II. Virus-cell interactions. - In a growing plaque, there are three species. Their interactions can be summarized by the reactions

$$
V+B \rightarrow^{k_{1}} I \rightarrow^{k_{2}} Y \cdot V
$$

where $Y$ is the production (or yield) of new viruses $(V)$ per infected host $(I), k_{1}$ is the rate constant of adsorption of viruses $(V)$ to uninfected bacteria $(B)$, and $k_{2}$ is the rate constant for death (or lysis) of infected bacteria $(I)$.

Instead of presenting the equations of our model directly, it will be much clearer to introduce them after considering several very simple situations.

Consider first a homogeneous medium, composed initially of infected cells $(I)$ and a few free viruses. As time goes on, cells will die and viruses $(V)$ will appear at a rate determined by the second of the reactions (1). This process can be modeled by means of the usual logistic equations,

$$
[V]_{t}=-Y[I]_{t}=Y k_{2}[I]\left(1-\frac{[I]}{[I]_{\max }}\right),
$$

where $[\cdots]$ denotes concentration, and the subindex $[\cdots]_{t} \equiv \partial / \partial t$ stands for the time derivative. The solution to Eqs. (2) under the appropriate boundary conditions $\left(\lim _{[I] \rightarrow[I]_{\max }}[V]=0\right.$ and $\left.\lim _{[I] \rightarrow 0}[V]=[V]_{\max }\right)$ is easily found,

$$
[V]=\frac{Y[I]_{\max }}{1+c_{1} \exp \left(-k_{2} t\right)},
$$

which describes well the corresponding experimental data and makes it possible to determine the values of $k_{2}$ and the yield $Y$ (see Fig. 1). $c_{1}$ is an integration constant which depends on the arbitrary definition of the moment $t=0$.

Another very simple case corresponds to the homogeneous, nonreproductive adsorption of viruses [first reaction in (1)]. Then, the usual equations are

$$
[V]_{t}=[B]_{t}=-k_{1}[V][B]=-[I]_{t},
$$




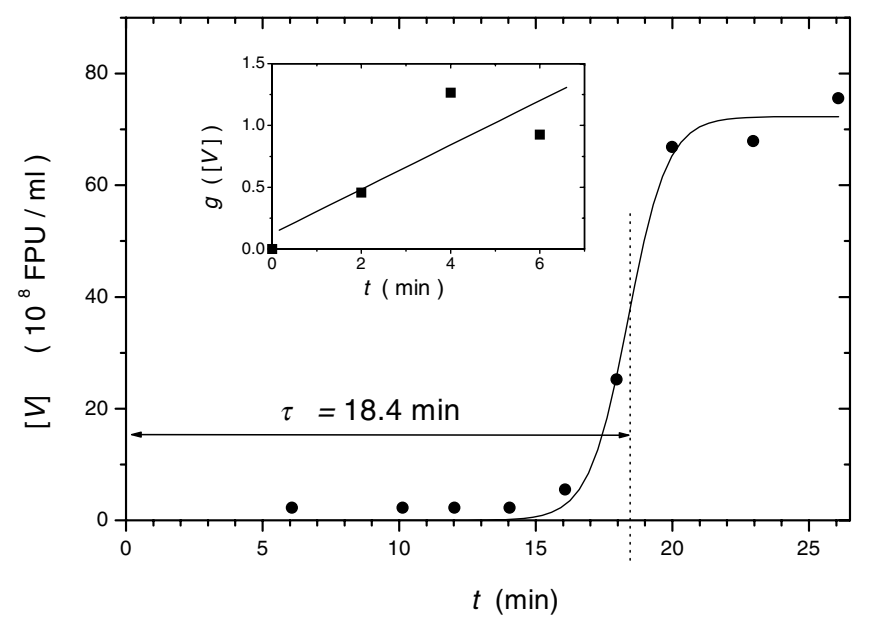

FIG. 1. One-step growth of virus T7 on E. coli versus the time elapsed after adsorption $(t=0)$. In this experiment, the concentration is uniform and adsorption is negligible $\left([B]_{t} \approx 0\right)$, attained by dilution. Full line: least-square fit of the experimental data points [14] to Eq. (3), which yields $k_{2}=$ $1.39 \mathrm{~min}^{-1}$ and $\tau=18.4 \mathrm{~min}$. Before the rise in $[V]$, the experimental dots refer in fact to infected bacteria [19], and the yield is the ratio of the final value $[V]_{\max }$ to this initial concentration [19], in this case $Y=34.5$. Inset: T7 adsorption on E. coli, based on the data in Ref. [20]. The assay was carried out in the presence of $\mathrm{KCN}$, which is known to inhibit the virus reproduction. Then Eqs. (4) hold; thus, $[B]=[V]+$ const. From the initial concentrations (taking care of the multiplicity) in Ref. [20], we find const $=1.39 \times 10^{8} \mathrm{ml}^{-1}$. The solution for $[V] \quad$ satisfies $\quad g([V]) \equiv \ln \{([V]+$ const $) /[V]\}-\ln \left\{\left([V]_{t=0}+\right.\right.$ const) $\left./[V]_{t=0}\right\}=$ const $\times k_{1} t$. Full line: least-square fit of the experimental data [20] to this equation. The slope yields $k_{1}=$ $(1.29 \pm 0.59) \times 10^{-9} \mathrm{ml} / \mathrm{min}$. This value is rather uncertain, but it turns out that the predictions are essentially the same throughout this range of values for $k_{1}$ (Fig. 2).

which, comparing to the appropriate experiment, allow us to determine the value of $k_{1}$ (Fig. 1, inset).

III. Plaque growth. - Consider now a situation in which both adsorption and reproduction take place. If the virus concentration is not uniform, virus diffusion has also to be considered and the simplest generalization of Eqs. (2) and (4) is

$$
\left\{\begin{array}{l}
{[V]_{t}=D_{\text {eff }}[V]_{r r}+F_{V}([V],[B],[I])} \\
{[B]_{t}=F_{B}([V],[B],[I])} \\
{[I]_{t}=F_{I}([V],[B],[I])}
\end{array}\right.
$$

where $D_{\text {eff }}$ is the effective diffusion coefficient (explained in detail in Sec. V), $[\cdots]_{r r} \equiv \partial^{2} / \partial r^{2}$ stands for the second spatial derivative in the radial direction from the plaque center, and

$$
\left\{\begin{array}{l}
F_{V}([V],[B],[I]) \equiv-k_{1}[V][B]+Y k_{2}[I]\left(1-\frac{[I]}{[I]_{\max }}\right) \\
F_{B}([V],[B],[I]) \equiv-k_{1}[V][B] \\
F_{I}([V],[B],[I]) \equiv k_{1}[V][B]-k_{2}[I]\left(1-\frac{[I]}{[I]_{\max }}\right) .
\end{array}\right.
$$

$178101-2$
IV. Time-delayed diffusion: approximate treatment.Equations (5) are Yin's classical model [16-18] of plaque growth with logistic dynamics. However, this model is unable to explain experiments [16-18]. Our idea is very simple. We will modify the classical model above by taking into account that there is a delay time between the moment when a virus adsorbs into a cell and that in which the cell dies and the new generation of viruses begins to spread. Therefore, in agreement with the derivation in Ref. [6], we propose to take into account this delay in the diffusion process by modifying the first of Eqs. (5) by its corresponding hyperbolic generalization, namely [6],

$$
[V]_{t}+\frac{\tau}{2}[V]_{t t}=D_{\text {eff }}[V]_{r r}+F_{V}+\frac{\tau}{2}\left(F_{V}\right)_{t}
$$
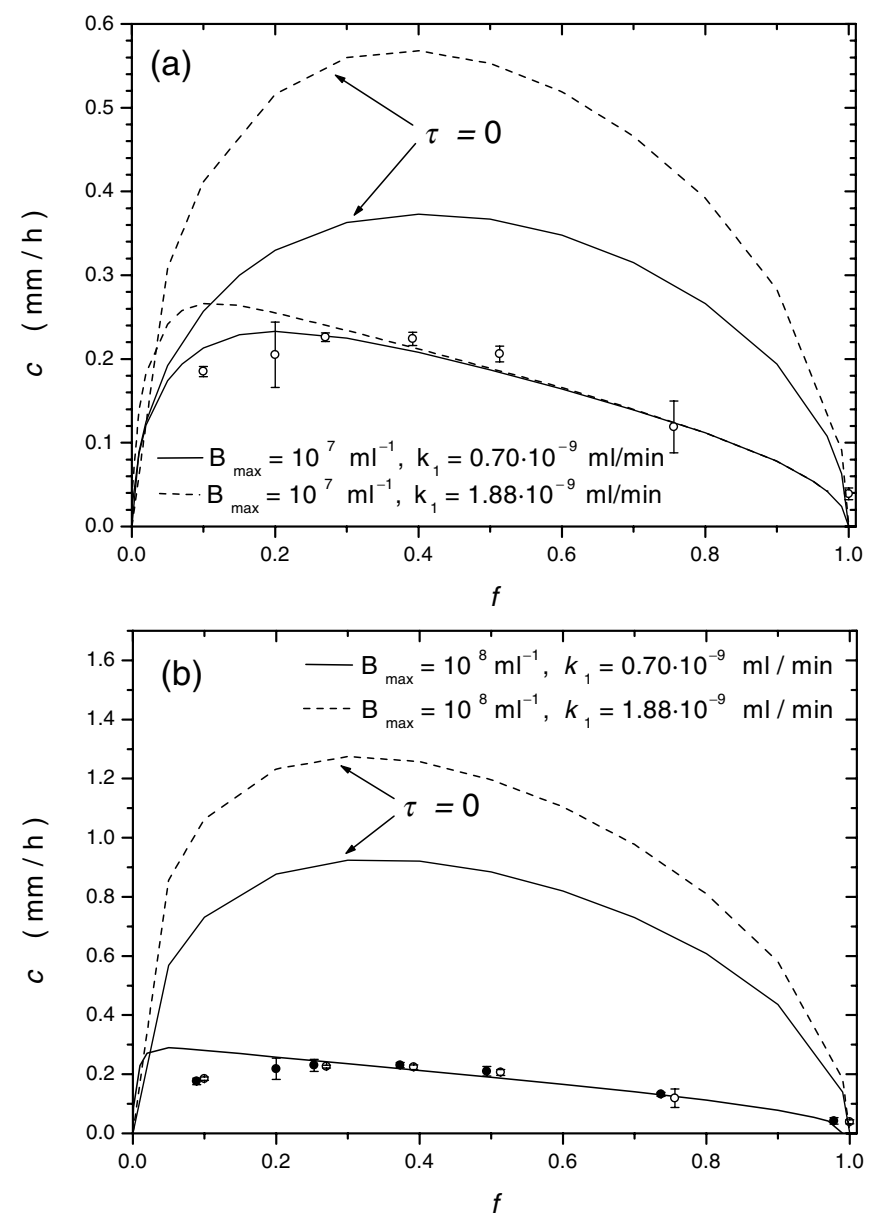

FIG. 2. Speed of the growth of T7 virus plaques on E. coli as a function of the bacterial relative concentration $f$. Upper curves: predictions of the classical model [17], i.e., Eqs. (7) with $\tau=0$. Lower curves: time-delayed model ( $\tau=18.4 \mathrm{~min}$, from Fig. 1). Symbols: experimental data [17]. The open and closed data symbols refer to initial bacterial concentrations of $10^{7}$ and $10^{8} \mathrm{ml}^{-1}$, respectively. The mean value and error for $f=0.2$ has been computed by taking into account that there are some additional experimental data available for $f=0.2$ in Refs. [14,15]. In (b), the two lower curves are not distinguishable from each other at this scale, showing that the uncertainty in the value of $k_{1}$ (Fig. 1, inset) has very little effect on the predicted speed. 
We stress that, for the moment, we are building the simplest possible model with a time delay by considering the wellknown hyperbolic approximation to the full time-delayed evolution equation (see "note added" at the end of this Letter). In the simple, hyperbolic approximation considered, our model is

$$
\begin{gathered}
{[V]_{t}+\frac{\tau}{2}[V]_{t t}=D_{\text {eff }}[V]_{r r}-k_{1}\left\{[V][B]+\frac{\tau}{2}([V][B])_{t}\right\}+Y k_{2}\left\{[I]\left(1-\frac{[I]}{[I]_{\max }}\right)+\frac{\tau}{2}\left[[I]\left(1-\frac{[I]}{[I]_{\max }}\right)\right]_{t}\right\},} \\
{[B]_{t}=-k_{1}[V][B], \quad[I]_{t}=k_{1}[V][B]-k_{2}[I]\left(1-\frac{[I]}{[I]_{\max }}\right) .}
\end{gathered}
$$

In the first of Eqs. (7), $\tau$ is the rest time, i.e., the time interval during which particles (viruses in our case) do not move [see Ref. [6] for the microscopic derivation of Eq. (6), including the factor $\frac{1}{2}$ appearing in its terms with $\tau]$. Therefore, in the case considered here, $\tau$ is simply the mean time interval from the adsorption of a virus by an uninfected host until the death of the thus infected cell. Thus, it may be inferred from Fig. 1 to be $\tau=18.4 \mathrm{~min}$. Note that the interpretation of the delay time above is analogous to that in the single-species, time-delayed theory of the Neolithic transition in Europe, where $\tau$ is the time interval between two successive migrations [6].

Let $B_{0}$ stand for the initial concentration of bacteria, previous to the arrival of viruses. We introduce the dimensionless variables $\bar{B} \equiv[B] / B_{0}, \bar{V} \equiv[V] / B_{0}, \bar{I} \equiv[I] /$ $B_{0}, \bar{t} \equiv k_{2} t, \bar{\tau} \equiv k_{2} \tau, \bar{r} \equiv r \sqrt{k_{2} / D_{\text {eff }}}$, and $\kappa_{1} \equiv k_{1} B_{0} / k_{2}$. We look for front solutions [17,21] by assuming the concentrations depend only on $\bar{z} \equiv \bar{r}-\bar{c} \bar{t}$, where $\bar{c} \equiv c / \sqrt{D_{\text {eff }} k_{2}}$ and $c$ is the front speed, and linearizing the system (7) in the wave-front edge $z \rightarrow \infty,(\bar{V}, \bar{B}, \bar{I})=\left(\varepsilon_{V}, 1-\varepsilon_{B}, \varepsilon_{I}\right) \approx$ $(0,1,0)$, with $\varepsilon \equiv\left(\varepsilon_{V}, \varepsilon_{B}, \varepsilon_{I}\right)=\varepsilon_{0} \exp (-\lambda z)$. For a solution $\left(\varepsilon_{V}, \varepsilon_{B}, \varepsilon_{I}\right) \neq(0,0,0)$ to exist, the determinant of the matrix corresponding to the linearized form of Eqs. (7) must vanish. This yields the characteristic equation for our problem, namely,

$$
\begin{aligned}
0= & \left(-1+\frac{\bar{\tau}}{2} \bar{c}^{2}\right) \bar{c} \lambda^{3}+\left\{-1+\left[1+\left(\kappa_{1}+1\right) \frac{\bar{\tau}}{2}\right] \bar{c}^{2}\right\} \lambda^{2} \\
& +\left\{\kappa_{1} \bar{c}\left[1-\frac{\bar{\tau}}{2}(Y-1)\right]+\bar{c}\right\} \lambda-\kappa_{1}(Y-1) .
\end{aligned}
$$

If the values of the parameters are known, this equation can be solved numerically in order to find out the dimensionless front speed $\bar{c}$. Numerical simulations [22] for a variety of cases have shown that the front travels with the minimum possible speed. Therefore, the minimum speed is calculated from $\bar{c}=\min _{\lambda>0}[\bar{c}(\lambda)]$, where $\bar{c}(\lambda)$ is given by characteristic Eq. (8). This is the marginal stability or linearization speed selection mechanism, which applies to the so-called pulled fronts [23,24].

$V$. Hindered diffusion. - We now have a time-delayed model and the values of the reactive parameters. Concerning diffusion, the effective coefficient for a solute (viruses in our case) dispersing through a continuous medium (agar) in the presence of a suspension of spheroids (host bacteria) which adsorb the solute is given by Fricke's equation [25],

$$
D_{\text {eff }}=\frac{1-f}{1+\frac{f}{x}} D
$$

where $f=B_{0} / B_{\max }$ is the concentration of bacteria relative to its maximum possible value. In the experiments $[14,15,17], f$ was measured indirectly by noting that the bacterial concentration $B_{0}$ (and thus $B_{\max }$ ) depends on the initial nutrient concentration, and typical bacterial concentrations were estimated in the range $10^{7}-10^{8} \mathrm{ml}^{-1}$ [17]. In Eq. (9), $D$ is the diffusion coefficient in the absence of suspended particles, and $x$ takes care of their shape according to

$$
x=\frac{-1}{1-\frac{1}{3}\left(\frac{2}{1-\frac{M}{2}}+\frac{1}{M}\right)},
$$

where

$$
M=\frac{1}{\sin ^{2} \phi^{\prime}}-\frac{1}{2} \frac{\cos ^{2} \phi^{\prime}}{\sin ^{3} \phi^{\prime}} \ln \left(\frac{1+\sin \phi^{\prime}}{1-\sin \phi^{\prime}}\right),
$$

and $\cos \phi^{\prime}=b / a$, with $a$ the dimension of the suspended spheroids (cells) along their axis of symmetry and $b$ their size along the other axis. Fricke's results have been found to agree very well with experimental observations of blood cell suspensions [25,26]. For spherical particles $(a=b), x=2$ and one recovers Maxwell's formula [27], which is the approximation previously used in virus diffusion by some authors $[16,17]$. For $E$. coli, $a / b \approx 4.0$ [28], which yields $x=1.67$, and we use this more accurate value. Also in Eq. (9), $D$ is the diffusion coefficient of the virus through agar in the absence of bacteria, which can be approximated to that of P22 [17] because it is very similar to T7 in size and shape [29], i.e., $D=$ $4 \times 10^{-8} \mathrm{~cm}^{2} / \mathrm{s}$ [30]. Now that we have estimations of the values for all of the parameters in the model $\left(Y, k_{2}, \tau\right.$, $k_{1}, B_{\max }, x$, and $D$ ) from independent experiments, we can compare the predictions both from our new, time-delayed model $(\tau=18.4 \mathrm{~min})$ and from the classical one $(\tau=0)$ to the observed infection speeds, without making use of any free or adjustable parameters.

VI. Theory versus experiment. - They are compared in Fig. 2. The classical approach (upper curves in Fig. 2) predicts speeds that are clearly inconsistent with the observed ones, as previously noted [16-18]. In contrast, the new time-delayed model (lower curves in Fig. 2) yields good quantitative agreement with the observations. This is the first time that the time-delay effect is used to explain wave-front propagation experiments that can be reproduced under controlled conditions.

VII. Conclusions. - Our model provides a satisfactory explanation for the growth of virus plaques. This is a basic problem which is also important because it provides an alternative way to predict and recognize virus strains, 
as well as their evolution [14,15]. Previous attempts to use reaction-diffusion models were unsuccessful. This led to the misbelief that physical models cannot explain these experiments [17,31]. Agreement to experiment has been attained here, after taking proper care of (i) the role of the delay time, (ii) the fact that the volume occupied by cells hinders the diffusion of viruses, and (iii) a careful reevaluation of the values of all of the parameters from independent experiments.

On the theoretical side, the present paper is the first one in which a system of reaction-diffusion equations, one of which is time delayed, has been derived and solved to obtain the front speed. The time-delayed approach reported should thus be useful in physical applications such as combustion [4] and superconducting [2] fronts, since the delay time arises naturally from the kinetic theory $[10,11]$. On the other hand, physicists can contribute to further topics related to that reported here, e.g., the characterization of mutant virus strains (which are recognized because of their front speeds $[13,14]$ ), the modeling of the front shapes in virus infections [15], etc.

The authors thank J. Yin for discussions, as well as J. Barbé, who also provided us with Ref. [20]. This work was supported in part by the Generalitat de Catalunya under Grant No. SGR-2001-00186, and by the MCYT under Grants No. BFM2000-0351 and No. REN20001621 CLI (J. F.).

Note added. - Our analysis above makes use of Eq. (6), which is in fact an approximation to the full time-delayed equation. It is an approximation because it keeps only a few terms in some Taylor expansions, e.g., $\bar{V}(r, t+\tau)-$ $\bar{V}(r, t) \simeq \tau \frac{\partial \bar{V}}{\partial t}+\frac{\tau^{2}}{2} \frac{\partial^{2} \bar{V}}{\partial t^{2}}[6,22]$. But since $[V]$ changes rapidly at a time scale smaller than $\tau$ (Fig. 1), this approximation may, in principle, break down. To see how this affects the results, we consider the full time-delayed equation, namely [see Ref. [32], Eqs. (16) and (21)],

$$
\begin{aligned}
{[V](r, t+\tau)-[V](r, t)=} & \sum_{k=1}^{\infty} \frac{\left(2 D_{\mathrm{eff}} \tau\right)^{k}}{(2 k) !} \frac{\partial^{2 k}[V]}{\partial r^{2 k}} \\
& +\sum_{k=1}^{\infty} \frac{\tau^{k}}{k !} \frac{\partial^{k-1} F_{V}}{\partial t^{k-1}}
\end{aligned}
$$

instead of its hyperbolic (or second-order) approximation (6). Then, it is easily seen by repeating the same steps as in Sec. IV that Eq. (8) is replaced by

$$
\begin{array}{r}
(\exp [\bar{c} \bar{\tau} \lambda]-1)\left[(1+\bar{c} \lambda)\left(\bar{c} \lambda+\kappa_{1}\right)-\kappa_{1} Y\right] \\
=[\cosh (\lambda \sqrt{2 \bar{\tau}})-1] \bar{c} \lambda(1+\bar{c} \lambda) .
\end{array}
$$

We have repeated the calculations leading to Fig. 2 by using this equation instead of (8), and the results change at most by a few percent (typically, below 1\%). The changes are so small that they cannot be seen in Fig. 2. This strengthens our conclusion that, for the first time, an experiment which (i) was previously unexplained and (ii) can be reproduced in the lab, has been explained in terms of time-delayed wave-front propagation.

[1] R. Kupferman, O. Shochet, E. Ben-Jacob, and Z. Schuss, Phys. Rev. B 46, 16045 (1992).

[2] S. J. Di Bartolo and A.T. Dorsey, Phys. Rev. Lett. 77, 4442 (1996).

[3] W. van Saarloos, M. van Hecke, and R. Holyst, Phys. Rev. E 52, 1773 (1995).

[4] J. Warnatz, U. Maas, and R.W. Dibble, Combustion (Springer, Berlin, 2001).

[5] O. Jousson et al., Nature (London) 408, 157 (2000).

[6] J. Fort and V. Méndez, Phys. Rev. Lett. 82, 867 (1999).

[7] P. Menozzi, A. Piazza, and L. L. Cavalli-Sforza, Science 201, 786 (1978).

[8] Y. Lee and J. Yin, Nature Biotech. 14, 491 (1996).

[9] V. Méndez, Phys. Rev. E 57, 3622 (1998).

[10] G. Chen, Phys. Rev. Lett. 86, 2297 (2001).

[11] D. Jou, J. Casas-Vázquez, and G. Lebon, Extended Irreversible Thermodynamics (Springer, Berlin, 2001).

[12] P. K. Galenko and D. A. Danilov, J. Cryst. Growth 216, 512 (2000); Phys. Lett. A 272, 207 (2000).

[13] Y. Lee and J. Yin, Nature Biotech. 14, 491 (1996).

[14] J. Yin, J. Bacteriol. 175, 1272 (1993).

[15] J. Yin, Biochem. Biophys. Res. Commun. 174, 1009 (1991).

[16] L. You and J. Yin, J. Theor. Biol. 200, 365 (1999).

[17] J. Yin and J. S. McCaskill, Biophys. J. 61, 1540 (1992).

[18] J. Fort, J. Theor. Biol. 214, 515 (2002).

[19] M. Delbrück, Biol. Rev. Proc. Cambridge Philos. Soc. 21, 30 (1946).

[20] K. Shishido, A. Watarai, S. Naito, and T. Ando, J. Antibiot. (Tokyo) 28, 676 (1975).

[21] J. D. Murray, Mathematical Biology (Springer, Berlin, 1993).

[22] V. Méndez, J. Fort, and J. Farjas, Phys. Rev. E 60, 5231 (1999).

[23] G. Dee and J. S. Langer, Phys. Rev. Lett. 50, 383 (1978).

[24] U. Ebert and W. van Saarloos, Physica (Amsterdam) 146D, 1 (2000).

[25] H. Fricke, Phys. Rev. 24, 575 (1924).

[26] J. Crank, The Mathematics of Diffusion (Oxford University Press, Oxford, 1975), 2nd ed., p. 271.

[27] J. C. Maxwell, A Treatise on Electricity and Magnetism (Dover, New York, 1954), Vol. 1, p. 440.

[28] T. D. Brock and M.T. Madigan, Biology of Microorganisms (Prentice-Hall, Englewood Cliffs, NJ, 1991), 6th ed., p. 40.

[29] D. Stollar and L. Levine, Methods Enzymol. 6, 848 (1963).

[30] H.-W. Ackermann, Path. Biol. 24, 359 (1976).

[31] J. Yin (personal communication).

[32] J. Fort and V. Méndez, Phys. Rev. E 60, 5894 (1999), Eqs. (16) and (21). 